\title{
FROM PLANETOIDS TO PLANETS
}

\author{
P. Barge and R. Pellat
}

\section{Introduction}

A common origin of the sun and the planets from the collapse of interstellar gas is now widely accepted. Regardless of how stars form, which is considered as the previous step of the whole story, the starting point is a flattened rotating cloud containing a mixture of dusts and gas (the so called Kant-Laplace Nebula). On the other hand the observations of young solar-mass stars show with increasing evidence that the gas is dispersed away on a time scale less than $10^{7}$ years and this provides us with a clear time constraint for model building since the formation of the giant gaseous planets have to take place on a shorter time scale.

Two classes of models can be distinguished following the mass of the Nebula is assumed to be of the order of or much smaller than one solar mass.

- In the case of a massive and turbulent Nebula the protosun has only a small fraction of its present mass and has not achieved its formation. In this accretion disk massive fragments would result from the gravitational instability and would lose a great part of their mass before to evolve into planets. This is the outline of the "giant gaseous protoplanets" model of Kuiper (1951), Cameron (1978) and others. These protoplanets would spiral inward as the excess of mass flows past them as the sun achieves its accretion and, when they reached the inner regions of the solar system, could have their gaseous envelope thermally or tidally stripped away; the remaining non volatile cores would then become the terrestrial planets. This model has the advantage to form the planets in a short time scale (some $10^{5} \mathrm{yrs}$ ), while the sun completes its accretion, but is faced with a number of difficulties:

- it requires a good timing of rather uncertain events (Wetherill, 1978);

- in the inner solar system, tidal instability is so important that the mass necessary to achieve stability is enormous and that no way to remove the resulting mass excesses is known at present (Wetherill, 1980; Ruskol, 1981);

- gravitational instability could be hardly compatible with the final feeding of the protosun (Lin and Papaloizou, 1980);

- the observed enhancement of the $\mathrm{C} / \mathrm{H}$ ratio of Jupiter with respect to solar value cannot be explained (Gautier and Owen, 1985).

Finally the difficulties encountered to explain the peculiar composition of Mercury and the existence of rocky cores into the interior of Jupiter and Saturn led Cameron (1988) 
to consider this scenario as a less promising one than the alternative possibility of planets formation through the sweeping-up of smaller bodies.

- In the case of a low mass Nebula the formation scenario is very different. The disk of gas is assumed to be stable against gravitational instability and formation of gaseous protoplanets; turbulence is weak and the temperatures remain on the average below the condensation temperature of the silicates. Then planets would grow by the gradual agglomeration of solid matter particles inside the cooling Nebula; this growth would proceed into two stages:

(i) the formation of planetoïds which occurs through a sticking of the dust grains as they sink toward the equatorial plane,

(ii) the accumulation of these planetoids during the collisional evolution of the swarm.

This is the outline of the classical accumulation model whose origin go back to the nineteenth century. Its prominence at present may be attributed to the pioneering works of the Moscow school (Safronov, 1969) and also to the Kyoto school (Hayashi et al, 1977).

In this Safronov's scenario the growth time scale of the terrestrial planets is of the order of $10^{7}-10^{8} y r s$ and the formation of the giant gaseous planets proceeds by the accretion of a solid core in some $10^{8} \mathrm{yrs}$ followed by the collapse of the surrouding gas (see for example Pollack, 1985). Such time scales were obtained from both analytic considerations (Safronov, 1969) and numerical simulations (Wetherill, 1980); they increase with increasing semi-major axis and become unrealistically large for the outer planets Uranus and Neptune.

This problem is generally circumvented by some a priori assumptions as for example a higher surface density or a larger focusing factor for the collisions (Greenberg et al, 1984; Lissauer, 1987) or again by assuming the pre-existence of some massive seeds (Safronov, 1969).

The assumption of an important gas drag, as in the model developed by the Kyoto school, can reduce the time scales somewhat but, in fact, only the so called "runaway accretion" described in a relatively few number of works (Greenberg et al, 1978; Wetherill and Stewart, 1989; Ohtsuki and Ida, 1990; Barge and Pellat, 1991) seems to eliminate the problem.

In this paper emphasis will be placed on these recent developments.

\section{The formation of the planetesimals}

The models developed by Safronov (1969) and by Goldreich and Ward (1973) start when the major part of the turbulent motions have decayed and that solid particles begin to condensate from the vapour phase of the cooling Nebula; they proceed into two stages.

- The first one corresponds to the separation of the solid and gaseous constituents. Due to the vertical component of the solar gravity and to the gas drag the dust grains spiral toward the equatorial plane and grow by condensation of solid vapour and collisional sticking. Safronov (1969) pointed out that large particles settle faster than smaller ones and, as they grow, can settle still faster in an accelerated way. In such a mechanism dust grains could reach a size of the order of a centimeter on a time scale of the order of $10^{3}$ years; the result would be a very thin dust layer in the equatorial plane of the Nebula.

- The second one corresponds to the onset of a gravitational instability inside the dust layer when the number density has become large enough. The layer break-up into a great number of self gravitating clumps which further collapse into compact bodies named "planetesimals". These primordial bodies would reach a characteristic size of the order of 10 $\mathrm{km}$ in some $10^{3}$ orbits (some $10^{9}$ planetesimals would be required to form the terrestrial planets).

In this widely accepted scenario the main rôle is played by the gravitational instability 
and any assumption on the difficult problem of the dust-grain sticking efficiencies is avoided. However a number of serious objections were raised by Weidenschilling (1989):

(i) the collisional sticking during dust grain sedimentation is more effective than previously believed and could even bypass gravitational instability completely (this comes from the fact that both the differential settling toward the central plane and the drag-induced collision rate inside the central plane can lead to a runaway growth);

(ii) the non keplerian rotation of the Nebula (induced by the pressure gradient) results in a shear between the gas and the dust layer which is a natural source of turbulence;

(iii) the solid bodies with a size less than one meter are likely sensitive to turbulent motions inside the Nebula (due to their coupling to the gas by the drag force) and, so, could not be affected by gravitational instabilities;

(iv) the actual sticking of solid dust grains is completely different from the assumption made in astronomy in which the problem reduces to simple coagulation of liquid drops. In fact aggregates of many smaller particles are fractal structures which are fluffy and very porous bodies. The coagulation and settling of these low-density structures seems to be much slower than in the "droplet" assumption because the smallest particles are consumed so rapidly that runaway growth by differential settling becomes uneffective.

Thus, if we keep in mind only gravitational instability, it is clear that we cannot see the forest for the trees. The formation of the planetesimals is faced with numerous problems; it seems to depend on a crucial way on the stickiness of the particles and on the local level of the turbulence inside the Nebula. So, it is likely that planetoïds did not appear suddenly with a uniform size as described in the classical scenario.

In fact whether planetoïd formation proceeds by a composite process involving sticking and gravitational instability or by sticking only remains, at present, a very controversial question. Nevertheless it is likely that one way or another a great number of planetoïds with size around some kilometers were formed.

The mass spectrum of these primordial bodies, which is the starting point of the following accumulation stage, is obviously very uncertain. Greenberg et al (1984) have suggested that a size distribution is more probable than a unique characteristic size because large particles settle faster and can form a gravitationally unstable sublayer before the smaller particles. The most frequent assumption is an exponential mass distribution but simple power laws cannot be rejected.

\section{The growth of the planetesimals}

Kilometer planetesimals are massive enough for gravitation to play the major role in the sticking and cohesion processes. On the other hand, the swarm of the planetesimals cannot be gravitationally unstable and the only possible way toward planet formation is the well known accumulation by collisional accretion which, in a number of recent works, was found to be faster than previously believed.

The idea of a runaway growth of the planetesimals appeared for the first time from the numerical simulations performed by Greenberg et al $(1978,1984)$; the growth, in a time scale of the order of $10^{4} \mathrm{yrs}$, was much more rapid than predicted by the analytical theory of Safronov. However the numerical procedure used by these authors has been suspected repeatedly (Wetherill and Stewart, 1989; Weidenschilling, 1989; Spaute et al, 1991) and, until now, it is not clear whether their results can be trusted or not.

The very large number of the primordial planetesimals (some $10^{10}$ bodies) led Safronov (1969) to use statistical gas dynamics and colloïdal coagulation theory to describe the growth of the planets. Thus the physical problem related to the accumulation of the 
planetesimals was set up clearly for the first time and reduced to find the evolutions of mass and velocity distributions.

- The evolution of the mass distribution is usually described with the help of the coagulation equation. On the one hand analytical solutions of this equation can be found only in some particular cases (Safronov, 1969) which are unappropriate to the study of the planetesimals; on the other hand numerical simulations are difficult and may lead to artificial accelerated growths (Ohtsuki et al, 1990). The recent work of Wetherill (1990) improves the understanding of this equation; it proves the existence of runaway solutions for appropriate form of the collision probabilities and provides a severe test for the accretion computations.

- The evolution of the velocity dispersions was faced with more or less drastic assumptions. Safronov simply assumes that the velocity dispersions keep step with the escape velocity of the largest bodies. Since then a wide variety of methods were used to improve the original Safronov's work (see the review of Stewart et al. 1984). From a two body kinetic approach of the problem Hornung et al (1985) derived concise expressions for the evolution of the velocity dispersions which were used later by Wetherill and Stewart (1989) to describe the accumulation of the planetesimals. The velocity dispersions of the various populations of planetesimals evolve under three competing processes:

- an energy loss due to sticking collisions and gas friction which results in a "cooling",

- a viscous stirring due to the kepler shear which results in a "heating",

- an energy equipartition which results from the dynamical friction and can lead either to a "heating" or to a "cooling" depending on the mass spectrum;

but, eventually, only viscous stirring and equipartition really dominate the evolution.

As long as the mass of the swarm is in the small bodies, the equipartition is the dominant contribution and the planetesimals can cool as they grow; then it results an increasing growth rate and, so, a stronger tendency toward equipartition. This is the runaway mechanism described by Wetherill and Stewart (1989) in which a single body emerges from the rest of the mass spectrum and keeps a small velocity dispersion (see figures 1 ). An attempt to incorporate high velocity fragmentation of the small bodies reduced the time scale somewhat but did not change their conclusions. The same kind of runaway was found in the recent numerical simulation performed by Spaute et al (1991).

However the three-body numerical integrations of orbital motions performed by Ida (1990) shows that dynamical friction and viscous stirring differ significantly from the twobody expressions when the relative velocities become approximately smaller than the Hill velocity $V_{H}=\Omega R_{H}$ (where $R_{H}$ is the well known Hill radius). Such a situation occurs at the beginning of the evolution; it implies that the two body formalism becomes unappropriate and that collisions and encounters must be described in a three-body formalism which accounts for the effect of the sun.

Due to this decreasing importance of the dynamical friction at the low velocity dispersions Barge and Pellat (1991) found that the cooling of the largest bodies is slowed down and that the low velocity runaway is inhibited. Thus, viscous stirring tends to dominate dynamical friction during the rest of evolution and the velocity dispersions of the largest bodies increase (as in the Safronov model), up even to values greater than $V_{M}$ (see figures 2); in contrast, at the end of the integration, these large bodies tend to runaway in an exponential way due to the increasing volume they sweep up (Barge and Pellat, 1991). This behavior of the velocity dispersion is obviously different from that of Wetherill and Stewart but the growth time scale of the largest bodies remains approximately the same. On the other hand the use of the three-body accretion rate derived by Greenzweig and Lissauer (1990), in place of the classical two-body expression, does not change these conclusions. 

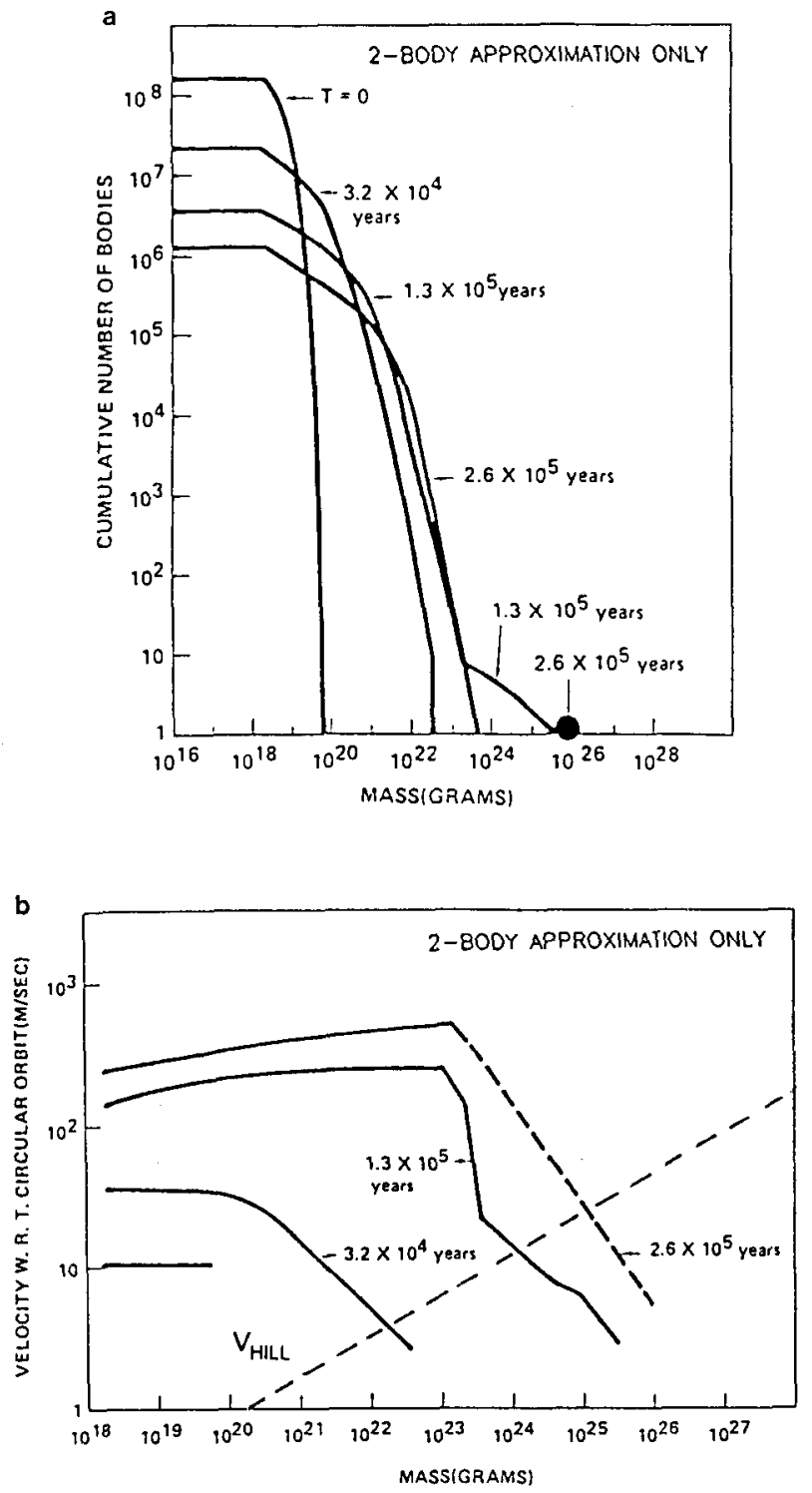

Figs.1. Evolution of the mass spectrum and the velocity distribution of the planetesimals. Initially the planetesimals are distributed between $0.99 \mathrm{AU}$ and $1.01 \mathrm{AU}$ and the mass spectrum is the exponential used by Safronov. The calculations are performed only in the two-body approximation and the surface density is $\sigma=22.4 \mathrm{gcm}^{-2}$ (after Wetherill and Stewart, 1989). (a) Evolution of the number of bodies. A single runaway body emerges from the swarm after $2.610^{5} \mathrm{yrs}$. (b) Evolution of the velocity dispersion. The velocity dispersions of the largest bodies remain always smaller than $V_{H i l l}$ that is in the domain in which three-body interactions come into play. 

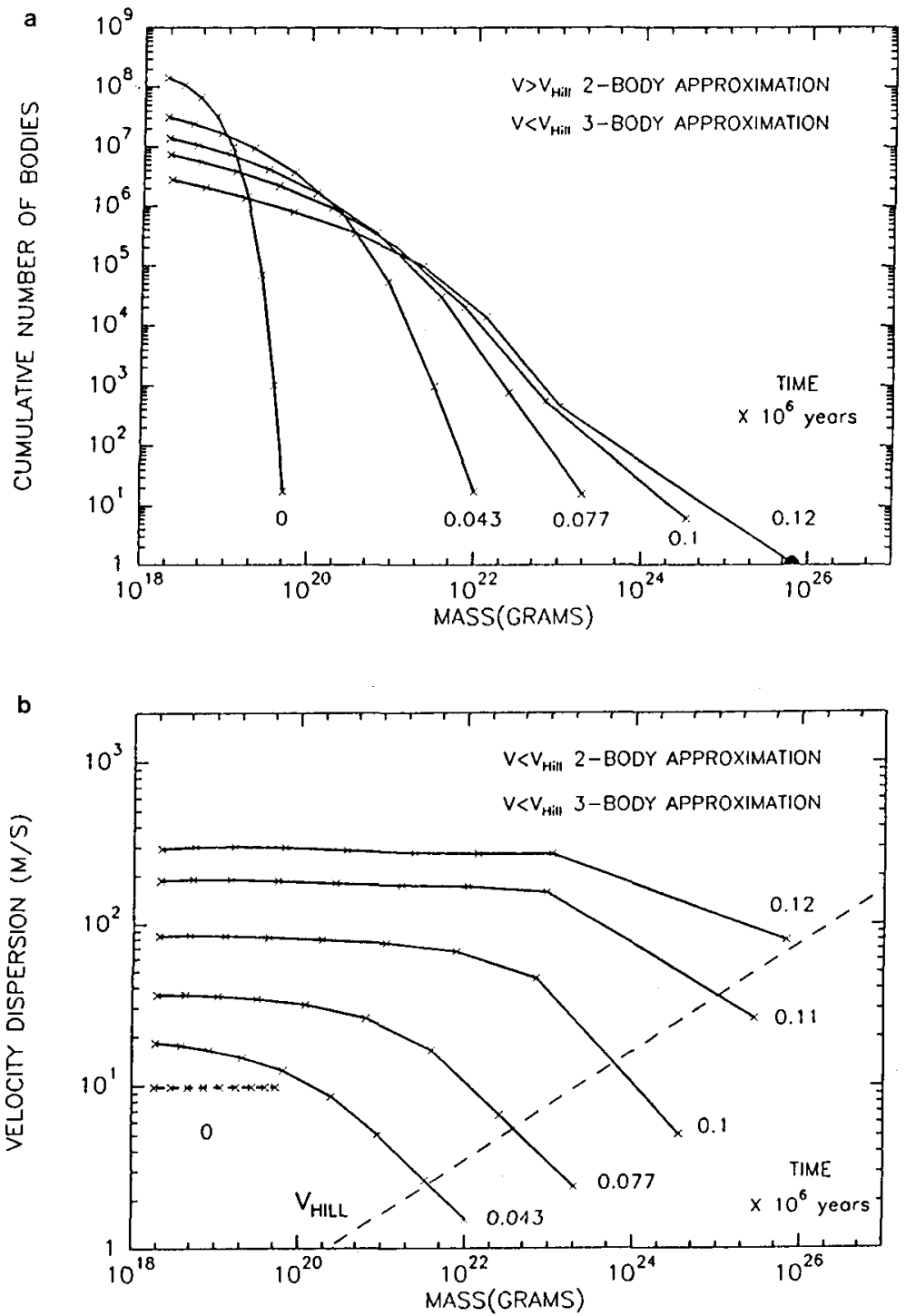

Figs.2. Evolution of the mass spectrum and the velocity distribution of the planelesimals. Initially the planetesimals are distributed between $0.99 \mathrm{AU}$ and $1.01 \mathrm{AU}$; the mass spectrum is the exponential used by Safronov and the surface density is $\sigma=22.4 \mathrm{gcm}^{-2}$. The calculations are performed with the account of three-body interactions when the velocity dispersions become approximately smaller than $V_{H i l l}$ (after Barge and Pellat, 1991). (a) Evolution of the number of bodies. Toward the end the largest bodies tend to runaway in an exponential regime. A single runaway body remains after $1.210^{5}$ yrs. (b) Evolution of the velocity dispersions. They increase since the beginning of the evolution and become greater than $V_{H i l l}$ toward the end. 
Finally the growth of a runaway body will end when its ring-shaped feeding zone has been depleted from all the smaller planetesimals. The resulting planetary embryos have masses which depend on the distance from the sun. Wetherill found that, in the region of the terrestrial planets, these embryos could reach masses of the order of $0.1 M_{\oplus}$ in a time scale of the order of $510^{4} \mathrm{yrs}$ whereas, in the Jupiter region, their masses would be $10 M_{\oplus}$ and the time scale $410^{5}$ yrs.

\section{The last stage of the accretion}

The final stage, during which the planetary embryos complete their growth to reach planet sizes, differs following the distance from the sun.

- In the region of the terrestrial planets the final scenario has been described in a qualitative way by the Moscow school (Safronov, 1969; Safronov and Ruzmaikina, 1985) and also with three-dimensional numerical simulations by Wetherill (1980) and by Cazenave et al. (1982). In a time scale of the order of $10^{7}$ to $10^{8}$ years, the simulations lead to four or six small planets (with approximately the mass of Mars) moving on elliptic orbits; the resulting exentricities are found to depend on the initial conditions. Starting from an excess of embryos in circular orbits Wetherill (1988) obtained the same kind of results.

- In the case of the giant planets the growth is complete only after the collapse of the nebular gas onto the cores (Bodenheimer and Pollack, 1986; Sekiya et al, 1987); that is roughly after less than $10^{5} \mathrm{yrs}$.

- In the region of the asteroid belt there is a strong mass depletion and a high relative velocity which exceeds the escape velocity of the largest asteroids. The origin of such velocities could be due to gravitational perturbations in relation with the presence of the outer giant planets. Wetherill and Stewart (1989) found that the velocity dispersion would be sufficiently strong to inhibit a low velocity runaway and that no planet can grow at this place; further, as collisional fragmentation promotes mass removal by gas drag and by entrainment in solar outflows, they estimate that, after $10^{6}$ years, the size distribution would be consistent with the present observations.

\section{Conclusions}

At present the most promising model of planet formation seems to be planet building by the accumulation of planetesimals; some authors (Wetherill, 1989) even refer to it as the "standard model".

Recent investigations of the problem, in which the mass and velocity distributions are studied simultaneously, show that the growth of the planetesimals proceeds through a runaway accretion and, so, that the formation of massive embryos can occur on a much shorter time scale than previously believed. This is particularly important in the region of the giant planets since the rapid growth of massive cores can trigger the collapse and the formation of the giant gaseous planets in a time scale compatible with the dissipation of the Nebula. Thus one of the most severe constraint on the formation of the solar system could be satisfied.

On the other hand the last stage of the formation of the terrestrial planets from the merging of planetary embryos remains very long (of the order of $10^{8} \mathrm{yrs}$ ), so that the embryos have to survive fragmentation and removal of matter throughout this period. At the end if a massive protoplanet cohabits with a swarm of planetesimals more complications 
appear in relation with the excitation of density wave pertubations, as for example the radial migration of the embryos and the associated gap formation inside the disk of planetesimals (Ward and Hourigan, 1989).

Further works and more observational constraints are needed to improve the models.

\section{References}

Barge, P., and R. Pellat (1990) Icarus 85, 481.

Barge,P., and R. Pellat (1991) Icarus 93, 270.

Bodenheimer, P. and J. B. Pollack (1986) Icarus 67, 391.

Cameron, A. G. W. (1978) in the Origin of the Solar System, ed. S.F. Dermott pp. 49-74. New York: Wiley, $668 \mathrm{pp}$.

Cameron, A. G. W. (1988) Annual Review of Astron. and Astrophys., 441.

Cazenave, A., B. Lago and K. Dominh (1982) Icarus 51, 133.

Gautier, D. and T. Owen (1985) in Protostars and Planets II, edited by D.C. Black and M. S.

Matthews. University of Arizona, Tucson p. 980.

Goldreich, P., and W. R. Ward (1973) Astrophys. J. 183, 1051.

Greenberg, R., J. F. Wacker, W. K. Hartmann, and C. R. Chapman (1978) Icarus 35, 1.

Greenberg, R., S. J. Weidenschilling, C. R. Chapman and D. R. Davis (1984) Icarus 59, 87.

Greenzweig, Y. and Lissauer, J.J. (1990) Icarus 87, 40.

Hayashi, C., K. Nakazawa, and I. Adachi (1977) Publ. Astron. Soc. Japan. 29, 163.

Hornung, P., R. Pellat and P. Barge (1985) Icarus 64, 295.

Ida, S. (1990) Icarus 88, 129.

Kuiper, G.P. (1951) Proc. Natl. Acad. Sci. USA 37, 383.

Lin, D. N. C. and J. Papaloizou (1985) in Protostars and Planets $I I$, edited by D.C. Black and

M. S. Matthews. University of Arizona, Tucson p. 980.

Lissauer, J. J. (1987) Icarus 69, 249.

Ohtsuki, K. and Ida, S. (1990) Icarus 85, 499.

Ohtsuki, K., Y. Nakagawa and K. Nakazawa (1990) Icarus 83, 205.

Pollack, J. B. (1985) in Protostars and Planets II, edited by D.C. Black and M. S. Matthews.

University of Arizona, Tucson p. 980.

Ruskol, E. L. (1981) Proc. Alpbach. Summer School ESA SP-164.

Safronov, V.S. (1969) Israel program for scientific translation, TT-F 677.

Safronov, V.S. and T.V. Ruzmaikina (1985) in Protostars and Planets II. (D.C. Black and M.S. Matthews, Eds.). Univ of Arizona Press, Tucson.

Sekiya, M., S. M. Miyama and C. Hayashi (1987) Earth Moon and Planets 39, 1.

Spaute, D., S.J. Weidenschilling, D.R. Davis, and F. Marzari (1991) Icarus 82, 147.

Stewart, G. R., D. N. C. Lin and P. Bodenheimer (1984) in Planetary Rings (R. Greenberg and A. Brahic, Eds.). Univ. of Arizona Press, Tucson.

Ward, W. R. and K. Hourigan (1986) Astrophys. J. 347, 490.

Weidenschilling, S. J., B. Donn P. Meakin (1989) in Proceedings of the Formation and Evolution of Planetary Systems Meeting. Eds. H.A. Weaver and L. Danly. Cambridge Univ. Press., 131.

Wethetill, G.W. (1980) The Continental Crust and its Mineral Deposit, pp. 3-24. Geol. Assoc., Canada, Waterloo.

Wetherill, G. W., and G. R. Stewart (1989) Icarus 77, 330.

Wetherill, G.W. (1989) in Proceedings of the Formation and Evolution of Planetary Systems

Meeting. Eds. H.A. Weaver and L. Danly. Cambridge Univ. Press., 1.

Wetherill, G.W. (1990) Icarus 88, 336. 